\title{
STUDIES OF THE FRONT END OF A NEUTRINO FACTORY*
}

\author{
William Fawley, Gregory Penn, Andrew Sessler, and Jonathan Wurtele \\ Lawrence Berkeley National Laboratory, Berkeley, CA 94720
}

\begin{abstract}
A neutrino factory employs muons which are produced, collected, cooled, accelerated and then stored so that their eventual decay produces an intense neutrino beam. A general description may be found in the paper by Geer [S. Geer, Phys. Rev. D, 57, 1 (1998)], and two upcoming Comments on Nuclear and Particle Physics articles [S. Geer, "Future prospects for muon facilities", see http://www-mucool.fnal.gov/monotes/muc0154.ps; also A. M. Sessler, "Neutrino Factories: The Facility", http://www-mucool.fnal.gov/monotes/muc0155.pdf]. In this contribution, we use analytic and numerical tools to investigate the performance of the front end of a neutrino factory. This region starts just after the target and ends just prior to the recirculating accelerators. Extensive previous work has resulted in designs used in the Fermilab Study of 1999-2000 and the Brookhaven Study of 2000-2001. Here we explore variations away from these particular designs, seeking possible improvements in final muon output, risk reduction, and ultimate cost. Our studies include changes in the overall front end geometry through optimization of the induction linac design and variations of the $\mathrm{rf}$ frequency in the cooling channel acceleration, and initial exploration of the use of helical wiggler fields to increase the range of initial muon energies that are captured.
\end{abstract}

\section{INTRODUCTION}

Two recent studies $[1,2]$ have examined in some detail the performance of the front end of the neutrino factory based upon an intense muon source. The muons are created by pions which, in turn, result from the impact of a high average power proton beam on a target. The performance of the frontend is critical to the final flux of neutrinos at the detectors-the initial beam occupies a region of phase space that vastly exceeds the acceptance of conventional accelerators, either linacs or recirculating accelerators. The beam must be manipulated in both longitudinal and transverse phase space. The longitudinal manipulation, often referred to as phase rotation, uses a series of drift regions and induction acceleration regions to first create a correlation between axial position and particle energy and, second, to accelerate low-energy particles and decelerate high-energy particles. This, in effect, takes a pulse that is a few ns in duration and hundreds of $\mathrm{MeV}$ in energy spread and turns it into a pulse $60 \mathrm{~ns}$ long and $15 \mathrm{MeV}$ in energy spread. The pulse is then bunched in low-frequency rf, becoming a series of about 60 bunches. The transverse phase space occupied by these bunches is too large, or, stated more precisely,

\footnotetext{
* Work supported by the U.S. Department of Energy under Contract DE-AC03-76SF00098 and Grant DE-FG03-95ER40963
}

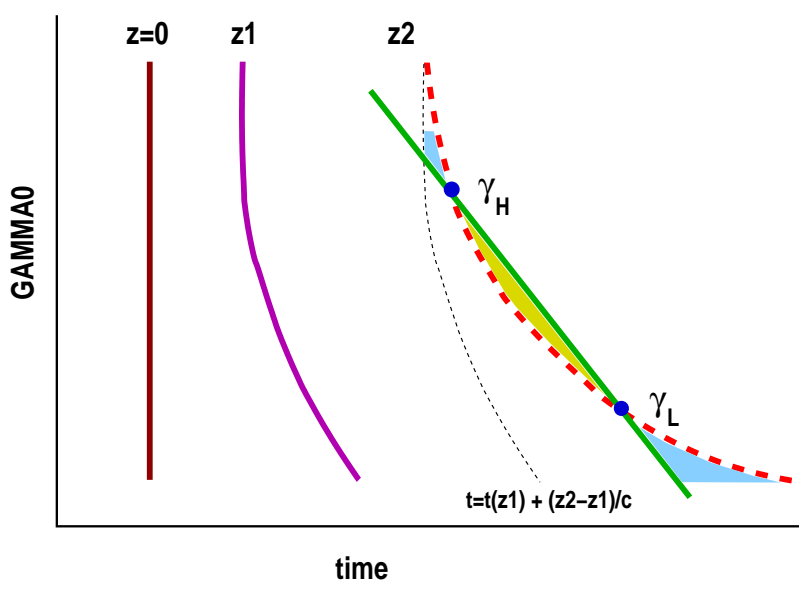

Figure 1: Symbolic representation of $\gamma(t, z)$ evolution. The green line represents the wanted $t_{2}\left(\gamma_{0}\right)$ relation; the dashed red line would occur without any acceleration at $z_{1}$ while the yellow (deceleration) and blue (acceleration) shaded areas indicate the necessary $\Delta t_{2}\left(z_{2}\right)$.

too few of the particles in these bunches are within the acceptance of the downstream system. The bunches are then cooled using ionization cooling. In this process the number of muons in the acceptance increases by about a factor of 3-5 [depending on the number chosen for the acceptance]. The larger acceptance, obviously, sees the smaller relative increase in the number of muons.

The cost of these frontend systems is roughly one third of the total cost of the factory complex. Since this part of the factory complex involves the most new technology, it would not be surprising to see greater impact of cost saving ideas here than in the more mature sections of the complex, such as the recirculating linac. The results reported here are preliminary, but indicate that analytical and numerical work may further improve the neutrino factory design. These studies also serve to provide an indication of the sensitivity of the design to parameter variations. For example, our study indicates performance is not strongly influenced by the choice of $\mathrm{rf}$ frequency between $150-300 \mathrm{MHz}$.

\section{PHASE ROTATION OPTIMIZATION}

The front end of a hypothetical muon facility has approximately $300 \mathrm{~m}$ of drift, induction accelerator cells, and "mini-cool" liquid hydrogen absorber sections. These sections help rotate a significant portion of the longitudinal phase space $(t, \gamma)$ from an originally nearly upright orientation at $z=0$ to a horizontal position with a sufficiently small $\delta \gamma$ to ensure a good trapping fraction in the downstream rf acceleration cells. R. Palmer[3] has proposed a 
"non-distorting" phase rotation scheme employing induction accelerator cells to minimize longitudinal phase space dilution (and thus the final $\sigma_{\gamma}$ ) during this rotation. One may divide induction cells into two groups. The first group at $z \approx z_{1}$ applies a time-varying energy kick $\Delta \gamma_{1}\left(t_{1}\right)$ whose value is chosen such that a significant portion of the muons arrives at a position $z_{2} \approx 300 \mathrm{~m}$ at a time $t_{2}$ that varies linearly with their original energy $\gamma_{0}$. Since the target pions (from which the muons decay) have a nearly constant density $d n\left(\gamma_{0}\right) / d \gamma_{0}$, the linear ramp in $t_{2}\left(\gamma_{0}\right)$ minimizes phase space dilution. The second group of cells at $z_{2}$ then applies a second energy kick $\Delta \gamma_{2}\left(t_{2}\right)$ such that the resultant beam energy $\gamma\left(t_{2}\right)$ is constant.

For simplicity, our analysis presumes that each induction cell group spans "zero" length in $z$. Then, through first order in $\gamma^{-2}$, we determine the required energy

$$
\gamma_{1}^{2} \equiv\left(\gamma_{0}+\Delta \gamma_{1}\right)^{2} \approx \frac{\gamma_{0}^{2}(1-\alpha)}{2 \gamma_{0}^{2} \zeta-\alpha}
$$

where $\alpha \equiv z_{1} / z_{2}, \zeta$ varies linearly with $\gamma_{0}$ ranging from $1 / 2 \gamma_{L}^{2}$ at $\gamma_{0}=\gamma_{L}$ to $1 / 2 \gamma_{H}^{2}$ at $\gamma_{0}=\gamma_{H}$, and $\gamma_{L, H}$ are energies for which $\Delta \gamma_{1} \equiv 0$ (see Fig.1). The exact choices of $z_{2}, \gamma_{L, H}$ and $\alpha$ are critical in determining the needed volt-seconds (and thus core material) in the induction cells, the value of $d t_{2} / d \gamma_{0}$ and, consequently, the minimum energy spread obtainable at $z_{2}$, and the useful range in $\gamma_{0}$ which can be successfully rotated. Lowering $\gamma_{L}$ increases $d t_{2} / d \gamma_{0}$ and decreases the accelerating volt-sec needed for $\gamma_{0} \leq \gamma_{L}$ but simultaneously increases the magnitude of the decelerating volt-sec which must be applied $\gamma_{L} \leq \gamma_{0} \leq \gamma_{H}$. Another consideration preventing $\gamma_{L}$ from becoming too small is that the resultant increase of $t_{2}\left(\gamma_{0}\right)$ and decreased $\gamma_{1}\left(\gamma_{0}\right)$ both increase the amount of muon decay between $z_{1}$ and $z_{2}$. Keeping $d t_{2} / d \gamma_{0}$ constant by moving $\gamma_{L}$ and $\gamma_{H}$ closer together reduces the required deceleration volt-seconds but simultaneously strongly increases the volt-seconds needed for acceleration for $\gamma_{0} \leq \gamma_{L}$. We are currently evaluating the optimum choice of $\left(z_{1}, z_{2}, \gamma_{L}, \gamma_{H}\right)$ applying these and other considerations.

\section{RF FREQUENCY SCALING}

The cooling channel used in the feasibility studies consists of a solenoidal focusing magnetic channel, liquid hydrogen absorbers, and rf to maintain bunching and replenish the muon energy lost in the absorber. The rf frequency was chosen so as to accommodate the large transverse beam size, and reduce scraping on apertures. Our main motivation for exploring different $\mathrm{rf}$ frequencies is to uncover the main factors which determine cooling channel performance, and to permit a more careful trade-off of performance with engineering costs. Some of these trade-offs are already evident. At higher frequencies, if the beryllium window radius is held fixed and the gradient increased (which a key motivation for the higher frequencies), there
Table 1: rf gradient and window thickness assumed at different frequencies

\begin{tabular}{|l|l|l|}
\hline Frequency & \multicolumn{2}{|c|}{ Gradient(MV/m) Window thick- } \\
& & ness $(\mathrm{mm})$ \\
$150 \mathrm{MHz}$ & 13.0 & 446 \\
$200 \mathrm{MHz}$ & 14.6 & 710 \\
$250 \mathrm{MHz}$ & 16.0 & 980 \\
$300 \mathrm{MHz}$ & 17.0 & 1200 \\
$300 \mathrm{MHz}$ & 17.0 & 840 \\
\hline
\end{tabular}

will be more heat dissipated in the window. Thus the window will need to be thicker, with a consequent increase in multiple scattering. To go to higher frequencies, effort will be needed to find ways of reducing the heating effect from multiple scattering, for example, by using tapered windows which are thinnest on axis. We can further increase our understanding of the beam dynamics issues that hurt performance. An example is the mismatch of the beam into the rf bucket. Even for an idealized bunching process, the beam becomes mismatched as it is cooled, and as the channel is altered. This is seen as oscillations in the rms longitudinal emittance and the energy-amplitude correlation.

The cooling channel used in the BNL Feasibility Study [2] can accommodate a range of rf frequency cavities without a change of the magnetic field structure. This allows us to make a preliminary comparison of the performance of various channels where the only change was to the rf parameters. Frequencies in the range $150-350 \mathrm{MHz}$ have been simulated with ICOOL [4].

The engineering assumptions used for the rf are determined by two factors: the electric fields which can reliably be generated in the rf cavity, and the heating which the beryllium windows are subjected to. The peak electric field on axis is assumed to scale as (frequency) $)^{0.4}$, while the heating on the beryllium window scales as (radius $)^{4}$ (frequency) ${ }^{1.75}$, with an additional factor related to saturation in the tangential fields, which grows linearly at small radius. The required beryllium thickness is proportional to the heating power on the windows. The total number of beryllium windows required also grows with $\mathrm{rf}$ frequency. The total power required for each MV of acceleration is roughly independent of rf frequency; however, for higher frequencies more acceleration is required due to the increase in beryllium.

The magnetic configuration of the cooling channel has been taken from Ref. [2]. This consists of a series of "Super-FOFO" lattices, each $2.75 \mathrm{~m}$ in length, which slowly vary to accentuate the point of maximum focus, then match into a similarly tapered series of $1.65 \mathrm{~m}$ lattices. The minimum of the beta function steadily decreases from 45 $\mathrm{cm}$ to $20 \mathrm{~cm}$.

The simulations were adjusted for varying rf frequency by changing the length of liquid hydrogen (LH) absorber to match the energy gain in the rf. The rf was tuned to be 60 degrees off crest. In addition, a muon bunch matched 


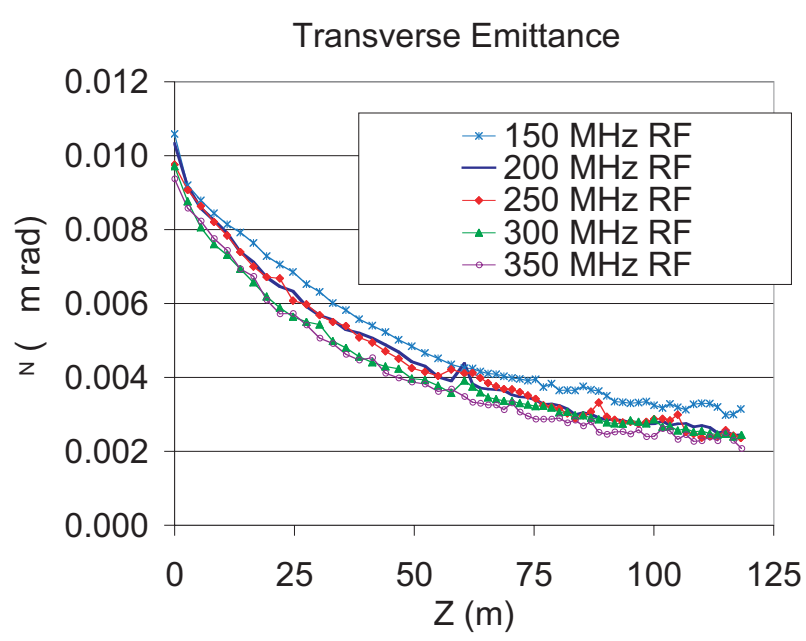

Figure 2: Normalized emittance as a function of propagation distance for various choices of rf frequency. The emittance is seen to be rather insensitive to the choice of frequency. The ICOOL [4] simulations have not been optimized for each frequency and all use an identical magnetic lattice. The results employed the post-processor ECALC9 [5].

into the rf was created for each frequency by propagating a beam in a channel devoid of material and with no apertures, having a magnetic field conforming to the first lattice type. The role of the LH absorbers was filled by a constant, decelerating electric field. The surviving particles were used as the initial input into the cooling channel. The initial beam properties are $\epsilon_{\perp} \simeq 10 \mathrm{~mm}, \sigma_{E} \simeq 18 \mathrm{MeV}$. The rms time spread corresponds to roughly 28 degrees of rf phase. The initial energy-amplitude correlation, defined as $E=E_{0}+C_{E} A$, where $A$ is the normalized transverse amplitude, is given by $C_{E} \simeq 0.25 \mathrm{GeV} / \mathrm{m}$.

In Fig. 2 the cooling of transverse emittance is plotted for various frequencies. It is clear that there is little sensitivity to the choice of frequency. The picture for the figure of merit, being the number of muons in the target phase space region, is more complicated. The figure of merit is fairly independent of rf frequency for the first 60 meters of cooling; after that, cooling saturates for frequencies above $200 \mathrm{MHz}$. For $150 \mathrm{MHz}$ rf, the cooling rate is slower and had not saturated by the end of the channel. The difference in performance is caused by the increasing number and thickness of beryllium windows for the rf cavities. When beryllium windows are removed from the simulations, channel performance is independent of frequency and only slightly better than the more realistic $200 \mathrm{MHz}$ results. Furthermore, the $200 \mathrm{MHz}$ case resulted from significant optimization of parameters; this optimization has yet to be performed for the other frequencies.

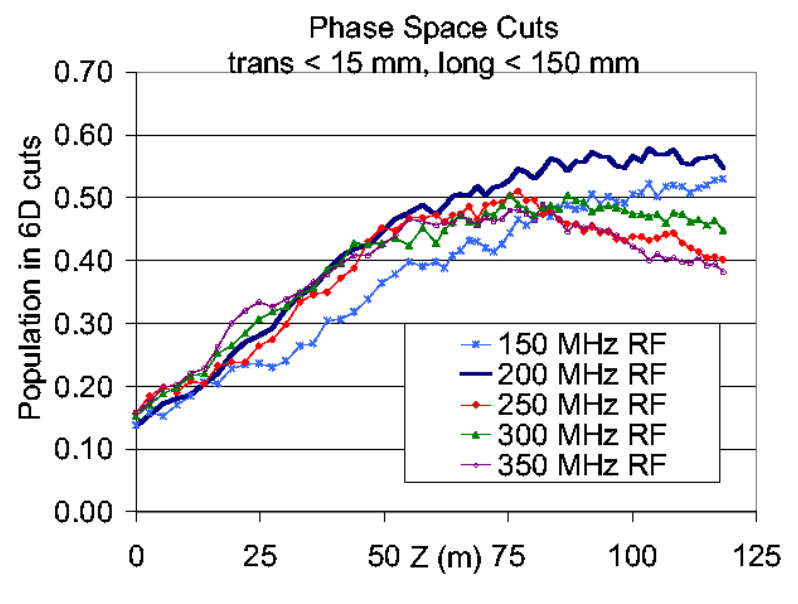

Figure 3: Performance of the cooling channel as function of propagation distance for various choices of rf frequency. The runs correspond to the emittances shown in the previous figure. Performance is measured as the number of muons in the six-dimensional acceptance of the downstream accelerator chain.

\section{CONCLUSIONS}

Two separate studies of modifications to the design of the frontend of the neutrino factory have been discussed. Analytical expressions have been obtained for the voltseconds required from an induction linac to compensate for the nonlinear distortions of the longitudinal phase space. A survey of $\mathrm{rf}$ frequencies shows that the design choice of $200 \mathrm{MHz}$ used in the Brookhaven and Fermilab studies is robust in the sense that variations of the frequency do not lead to reduced performance. We are hopeful that further investigations of the wide range of unexplored parameter space undertaken by ourselves and other members of the Neutrino Factory and Muon Collider Collaboration and by other groups worldwide will result in higher neutrino yield, and lower costs.

\section{REFERENCES}

[1] 'FNAL Feasibility Study on a Neutrino Source Based on a Muon Storage Ring", Holtkamp and Finley, Eds., FNAL Report available at http://www.fnal.gov/projects/muon_collider/reports.html]

[2] 'Feasibility Study of a Neutrino Factory II", Zisman and Ozaki, Eds, available at http://www.cap.bnl.gov/mumu/studyii/

[3] R. Palmer, "Non-Distorting Phase Rotation Frontends," Mucool Note 114, available at http://wwwmucool.fnal.gov/mcnotes/muc0114.ps.

[4] R. Fernow, "ICOOL, A SImulation Code for Ionization Cooling of Muon Beams," Proceedings of the 1999 Particle Accelerator Conference, A. Lucio and W. MacKay, eds. (IEEE, Piscataway, NJ, 1999) p. 3020.

[5] The ecalc9 code was written by G. Penn and is freely available. 